\title{
Electron Beam Current Loss at the High-Vacuum- High-Pressure Boundary in the Environmental Scanning Electron Microscope
}

\author{
Gerasimos D. Danilatos, ${ }^{1 \star}$ Matthew R. Phillips, ${ }^{2}$ and John V. Nailon ${ }^{3}$ \\ ${ }^{1}$ ESEM Research Laboratory, 98 Brighton Boulevard, North Bondi, New South Wales 2026, Australia \\ ${ }^{2}$ Microstructural Analysis Unit, University of Technology, Sydney, PO Box 123, Broadway, New South Wales 2007, Australia \\ ${ }^{3}$ Centre for Microscopy and Microanalysis, The University of Queensland, Brisbane, Queensland 4072, Australia
}

\begin{abstract}
A significant loss in electron probe current can occur before the electron beam enters the specimen chamber of an environmental scanning electron microscope (ESEM). This loss results from electron scattering in a gaseous jet formed inside and downstream (above) the pressure-limiting aperture (PLA), which separates the high-pressure and high-vacuum regions of the microscope. The electron beam loss above the PLA has been calculated for three different ESEMs, each with a different PLA geometry: an ElectroScan E3, a Philips XL30 ESEM, and a prototype instrument. The mass thickness of gas above the PLA in each case has been determined by Monte Carlo simulation of the gas density variation in the gas jet. It has been found that the PLA configurations used in the commercial instruments produce considerable loss in the electron probe current that dramatically degrades their performance at high chamber pressure and low accelerating voltage. These detrimental effects are minimized in the prototype instrument, which has an optimized thin-foil PLA design.
\end{abstract}

Key words: environmental scanning electron microscope, electron scattering, electron loss, differential pumping, gas jet, pressure limiting aperture, image noise, electron skirt

\section{INTRODUCTION}

The environmental scanning electron microscope (ESEM) has now become a standard commercial instrument and the comprehensive details of its operation can be found in two extensive works by Danilatos (1988, 1990). The specimen chamber of the microscope contains a gaseous environment, which allows all natural surfaces, wet or dry, insulating or conducting, to be viewed and analyzed. Present-day ESEMs use conventional differential pumping across a pres-

Received May 12, 2000; accepted February 21, 2001.

*Corresponding author sure-limiting aperture (PLA) to separate the high vacuum of the electron optics column from the high pressure of the specimen chamber. A transition region of intermediate pressures is established at the boundary between the two extreme pressure regions of the microscope. When the electron beam enters the transition region, it collides with gas molecules and suffers initial electron current loss above the PLA before it enters the specimen chamber. The loss of beam electrons in the transition region is an inevitable consequence of the differential pumping method used. The magnitude of the electron loss in the transition region is determined by the PLA design of a given instrument, which defines the spatial dimensions of the gas density distribu- 
tion in the inevitable gas plume that forms downstream ("above") of the PLA. For a given specimen chamber pressure and gas, the gaseous plume represents a certain mass thickness, which the electron beam must overcome before it enters the high-pressure region of the ESEM. Therefore, the optimum PLA design is one that minimizes the mass thickness of the gas plume and allows maximum transmission of the electron beam current.

An early experimental prototype ESEM was designed to operate with minimum electron beam current loss in the gas pressure transition region (Danilatos, 1981, 1983). With this microscope, the minimum beam current loss was experimentally determined to occur when (a) the wall thickness of the PLA was much less than its diameter and $(b)$ the conductance of the downstream evacuation pipe was as large as possible, which, together with sufficient pump speed, created a minimum back-pressure downstream of the transition region. In this prototype ESEM, commercially available copper apertures with diameters ranging from 50 to $600 \mu \mathrm{m}$ and a wall thickness of $10 \mu \mathrm{m}$ and $30 \mu \mathrm{m}$ were used as thin-walled PLAs. Danilatos (1983) has conducted a detailed experimental study of the gas dynamics of the supersonic gas jet that forms above these thin PLA apertures. The characteristic variation of gas density both below and above a thin PLA is reported elsewhere (Danilatos, 1991, 1993).

Optimization of the differential pumping system, together with the development of the appropriate imaging signal detection techniques, has allowed an ESEM to operate at any pressure between vacuum (such as that used in a conventional SEM) and one atmosphere (Danilatos, 1981, $1983,1985,1988)$. However, as chamber pressure is increased, the low magnification field of view decreases because smaller-diameter PLAs are required to sustain the larger pressure differential.

Different ESEM applications require different specimen chamber pressures, which can be divided into various functional or practical pressure ranges, such as (a) pressure sufficient to suppress charging effects, usually up to around $200 \mathrm{~Pa}$; (b) pressure sufficient to maintain liquid water phase, i.e., $>609 \mathrm{~Pa}$, corresponding to the saturation water vapor pressure at $273 \mathrm{~K}$ temperature; (c) pressure corresponding to the saturation water vapor pressure at room temperature, i.e., around $2 \mathrm{kPa}$; (d) saturation water vapor pressure at "living body" temperature $\left(36^{\circ} \mathrm{C}\right)$, i.e., around 6 $\mathrm{kPa}$; (e) gas pressure that sustains animal life, i.e., around 20-100 kPa; and $(f)$ an open-ended ESEM or atmospheric SEM, i.e., an instrument without a specimen chamber, ca- pable of operating at full atmospheric pressure for in situ examination of large objects (Danilatos, 1981, 1983, 1985, 1988).

In the present work, the gas density variation in the region downstream of the PLA was computed using the direct simulation Monte Carlo (DSMC) method (Bird, 1995). The computed gas density function was used to find the effective mass thickness of the gas jet and the fraction of electrons scattered out of the primary electron beam above the PLA. The oligo-scattering regime has been defined by Danilatos (1988) as the gas-pressure and working-distance range over which the focussed beam spot at the specimen plane retains more than $5 \%$ of the initial number of electrons in the vacuum. In this regime, the useful beam spot is surrounded by a very broad electron skirt formed by the scattered electrons. The electron skirt contributes to the background noise and does not degrade the spatial resolution of the image as long as sufficient current remains in the beam spot. However, the electron skirt can seriously degrade the spatial resolution in X-ray microanalysis (Bolon, 1991; Griffin, 1992; Doehne, 1997). The electron beam current loss and the formation of the accompanying electron skirt occurs primarily along the beam path between the PLA and the specimen in the specimen chamber. However, because the electron scattering begins prior to the beam entry into the specimen chamber, it is necessary to quantify these effects in order to determine their significance. Whereas the scattering of electrons in the specimen chamber for a given gas type and pressure depends on the working distance chosen by the instrument operator, the scattering that occurs above the PLA is characteristic of the gas dynamics for a given differential pumping design. The objective of this article is to report results on the characteristic gas density function and the electron beam current loss above the PLA for three different cases-namely, two commercial and one prototype ESEM instruments. This study was prompted by concerns that a significant deviation from expected imaging performance has been found in practice with the commercial ESEM instruments, especially at low accelerating voltage. We show here that the deterioration of imaging performance is due largely to nonoptimum geometric design of the PLA, resulting in unnecessary electron beam current loss above the PLA.

\section{Materials and Methods}

The properties of the gas flow through a PLA can be determined experimentally (Danilatos, 1983) or calculated from 
basic theory. However, the calculation is not always practical, because analytical functions of the gas flow exist only for the extreme cases of either continuum flow (i.e., at very high pressures or very large apertures) or free-molecule flow (i.e., at very low pressure or very small apertures) and only for limited geometries. The ESEM operates somewhere between these two extremes and therefore the optimum ESEM geometry is generally very difficult or impractical to solve, even when analytical solutions are applicable. Fortunately, the gas flow properties can be computed by the DSMC method (Bird, 1995).

The DSMC method is a technique for computer modelling the gas flow dynamics of a real gas by simulating some thousands, or even millions, of molecules at different gas pressures and different physical extents of the gas flow field. The velocities and positions of these molecules are stored in the computer and are modified with time as the molecules move and collide between and through the boundaries of the flow field. Once the entry and exit for a given gas are initially set, the program is allowed to run until it reaches a steady state, whereupon the program continues to sample the equilibrium properties until a satisfactory smooth average for each field point is achieved. The DSMC routines used in this work were initially developed for space engineering problems involving large space vehicles in rarefied gas conditions and required the use of mainframe computers. The availability of fast and inexpensive personal computers has allowed these routines to be adapted and used for gas flow computations in the ESEM (Danilatos, 1991, 1993). Along with the gas density, the simulation also determines the temperature, velocity, and Mach number at every point in the flow field, as well as the leak rate of gas through various interfaces defining the computational zones necessary to set up at the start of each routine; thus the leak rate through the various apertures can be readily obtained. In the present work, the gas flow dynamics of three different ESEM PLA differential pumping systems was simulated using the DSMC technique.

The first case involves a thin-edge PLA that can approximate the prototype ESEM situation. This PLA consists of a single $0.5-\mathrm{mm}$ diameter PLA on a $0.1-\mathrm{mm}$-thick plate, with the rim of the aperture tapered at a $45^{\circ}$ angle diverging in the downstream direction of the gas flow. The PLA geometry and the gas flow density contours are shown in Figure 1, where only half of the PLA cross section is drawn because the flow field is axially symmetric. The specimen chamber of the ESEM, located on the left side, is maintained at a given pressure, and the gas leaking through the aper-

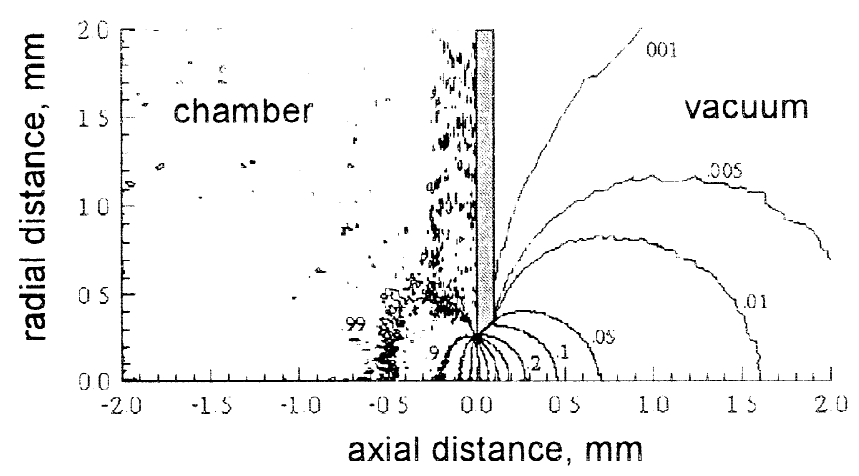

Figure 1. Density contours at constant $\times 2.47 \times 10^{22}$ atoms $/ \mathrm{m}^{3}$. Semi-cross section of thin-plate pressure-limiting aperture (PLA) with density contours of argon flowing from left (with specimen chamber pressure at $100 \mathrm{~Pa}$ ) to right (with pump at $0.00 \mathrm{~Pa})$.

ture is pumped out from the right side that is maintained at vacuum. The electron beam travels along the axis in the direction from low to high pressure (right to left).

The second case involves the PLA assembly of an ElectroScan E3 model ESEM (1991) located at the University of Queensland (QLD). The axially symmetric semi-cross section of PLA assembly is drawn in Figure 2, without gas flow contours for clarity. This assembly consists of primary and secondary PLA, and it is generally referred to as a "bullet." Gas leaking through the primary aperture is pumped out via a port located between the primary and secondary PLAs. The specimen chamber is located on the left side of the drawing, whereby gas enters from side CR and exits from opening IJ. Some gas also exits from the secondary PLA formed on plate LM. The electron beam travels from right to left along the axis QR.

The third case involves the PLA assembly employed in a Philips XL30 ESEM (1999) located at the University of Technology, Sydney (UTS). The axi-symmetrical cross section of the corresponding bullet assembly is drawn in Figure 3 , again without gas density contours. The specimen chamber is located on the left side of the drawing, whereby gas enters from side CU and exits from opening LM. Some gas also exits from the second PLA formed on plate OP. The electron beam travels from right to left along the axis TU.

The electron beam encounters gas molecules with which it collides well before it enters the specimen chamber because of the gas plume or jet forming downstream of (i.e., above) the PLA plane. The gas density decreases rapidly along the axis of the jet, but the precise variation of density depends on the geometry of the walls that constrain the gas. 


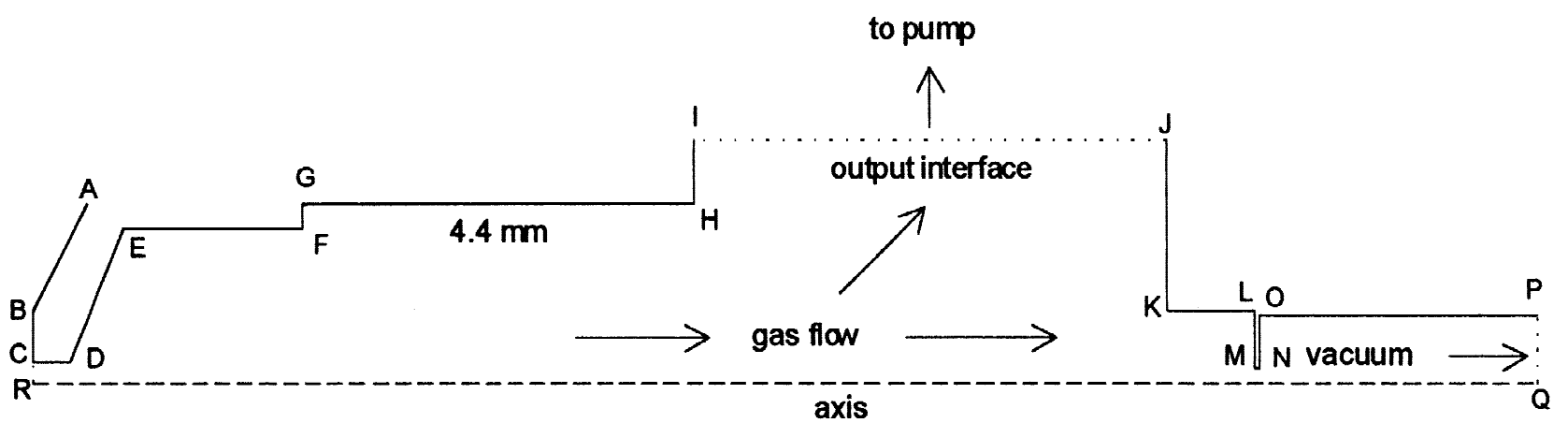

Figure 2. Scaled drawing of the geometry of the "bullet" assembly of the ElectroScan E3 environmental scanning electron microscope (ESEM) (1991), located at the University of Queensland, used for gas flow computation. Line RQ is the axis of symmetry,
$\mathrm{GH}=4.4 \mathrm{~mm}, \mathrm{ABC}$ faces the specimen chamber, CDEFGHI and JKLMNOP are the inside surfaces defining the gas flow, CR is the radius of the PLA, $\mathrm{IJ}$ is the output interface at pump pressure, and PQ is the interface with the column vacuum.

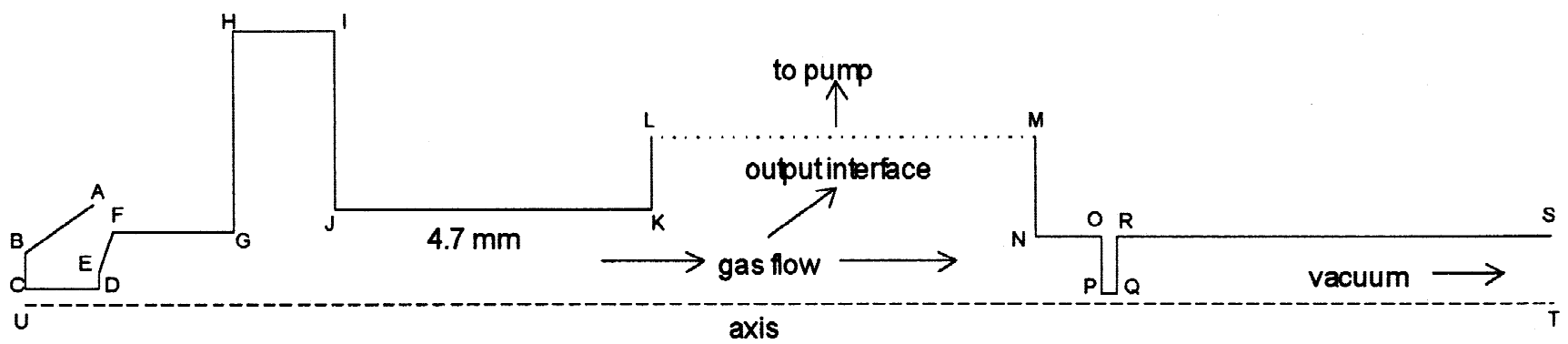

Figure 3. Scaled drawing of the geometry of the "bullet" assembly of the XL30 ESEM (1999), located at the University of Technology, Sydney, used for gas flow computation. Line UT is the axis of symmetry, $\mathrm{JK}=4.7 \mathrm{~mm}$, $\mathrm{ABC}$ faces the specimen chamber, $\mathrm{CDE}$ -

A quantitative evaluation of this phenomenon is necessary in order to establish the actual beam current loss above the PLA. This evaluation is achieved by finding the amount of electron scattering in the plume through the use of electron scattering theory (Danilatos, 1988).

Electron scattering theory can be used to quantitatively determine the loss in electron probe current in the gas jet above the PLA once the plume's gas density function has been simulated using the DSMC technique. The electron scattering process is governed by the Poisson distribution probability $P(x)$

$$
P(x)=\frac{m^{x} e^{-m}}{x !}
$$

which gives the probability for an electron to undergo $x$ number of collisions, when the average number of collisions
FGHIJKL and MNOPQRS are the inside surfaces defining the gas flow, CU is the radius of the PLA, LM is the output interface at pump pressure, and ST is the interface with the column vacuum.

per electron is $m$. Knowledge of the parameter $m$ allows us to immediately find the electron beam current $I$ that is transmitted completely without any scattering by the gas molecules, when the initial incident beam current in vacuum is $I_{0}$. The fraction of transmitted beam is given by the exponential equation

$$
\frac{I}{I_{0}}=e^{-m}
$$

The parameter $m$ is found from the gas density function $n(z)$ along the axis $z$ and the total scattering cross section $\sigma_{T}$ of the gas:

$$
m=\sigma_{T} \int n(z) d z
$$

The above integration is performed between any two limits that define the gas layer of interest. In the present work, the 


\begin{tabular}{lllllll}
\hline $\begin{array}{l}\text { Table } \\
\text { Aperture }\end{array}$ & Corresponding Values for a Thin Pressure-Limiting \\
\hline$p$ & $p_{1}$ & $\begin{array}{l}\text { mol_thick } \\
\text { ( } \begin{array}{l}\left.\text { (atoms } / \mathrm{m}^{2}\right) \\
(\mathrm{Pa})\end{array}\end{array}(\mathrm{eV})$ & $\sigma_{T}\left(\mathrm{~m}^{2}\right)$ & $m$ & $I / I_{0}$ \\
\hline 100 & 0 & $4.00 \mathrm{E}+18$ & 5000 & $1.56 \mathrm{E}-20$ & 0.06255 & 0.939 \\
100 & 0 & $4.00 \mathrm{E}+18$ & 10,000 & $8.28 \mathrm{E}-21$ & 0.03316 & 0.967 \\
100 & 0 & $4.00 \mathrm{E}+18$ & 15,000 & $5.73 \mathrm{E}-21$ & 0.02295 & 0.977 \\
100 & 0 & $4.00 \mathrm{E}+18$ & 20,000 & $4.43 \mathrm{E}-21$ & 0.01774 & 0.982 \\
1000 & 0 & $5.69 \mathrm{E}+19$ & 5000 & $1.56 \mathrm{E}-20$ & 0.88874 & 0.411 \\
1000 & 0 & $5.69 \mathrm{E}+19$ & 10,000 & $8.28 \mathrm{E}-21$ & 0.47111 & 0.624 \\
1000 & 0 & $5.69 \mathrm{E}+19$ & 15,000 & $5.73 \mathrm{E}-21$ & 0.32602 & 0.722 \\
1000 & 0 & $5.69 \mathrm{E}+19$ & 20,000 & $4.43 \mathrm{E}-21$ & 0.25206 & 0.777
\end{tabular}

$p$, specimen chamber pressure; $p_{1}$, pump pressure; mol_thick, molecular thickness; $E$, accelerating voltage; $\sigma_{T}$, total scattering cross section; $m$, average number of collisions; $I / I_{0}$, transmitted fraction of beam.

upper integration value was defined by a point where the gas density is essentially reduced to a negligible value and further integration adds $<0.5 \%$ to the total integration value. Using this criterion, the upper limit of integration point corresponded to $10 \mathrm{~mm}$ for the first case (thin PLA, Fig. 1), $13.7 \mathrm{~mm}$ for the second case (QLD, Fig. 2), and 16 $\mathrm{mm}$ for the third case (UTS, Fig. 3); the latter two upper limits corresponded to the location of the second PLA, because the pressure beyond those apertures was negligible. The lower integration limit was set to the gas entry plane of the first PLA located at the origin of the abscissa axis, although the entire computational flow field extended to -2 $\mathrm{mm}$ below the first PLA to account for the gas depletion zone immediately below the aperture. The integral term in equation (3) represents the molecular thickness (mol_thick), which is equivalent to the mass thickness (i.e., density $\times$ thickness as used in transmission electron microscopy work for thin specimen sections):

$$
\text { mol_thick }=\int n(z) d z
$$

Although this mathematical analysis is correct in absolute terms, the reliability of the beam current loss results determined in this work will ultimately depend on the accuracy of the total scattering cross section used in the calculation. Consequently, argon has been used as the test gas because its scattering cross section can be derived analytically as a monatomic gas (Jost and Kessler, 1963; Danilatos, 1988). The values used are tabulated for different accelerating voltages in Tables $1-3$.
Table 2. Corresponding Values for the ElectroScan E3 ${ }^{\mathrm{a}}$

\begin{tabular}{lccrccc}
\hline$p$ & $p_{1}$ & mol_thick & \multicolumn{1}{c}{$\mathrm{E}$} & & & \\
$(\mathrm{Pa})$ & $(\mathrm{Pa})$ & $\left(\right.$ atoms $\left./ \mathrm{m}^{2}\right)$ & \multicolumn{1}{c}{$(\mathrm{eV})$} & $\sigma_{T}\left(\mathrm{~m}^{2}\right)$ & $m$ & $I / I_{0}$ \\
\hline 100 & 0 & $9.70 \mathrm{E}+18$ & 5000 & $1.56 \mathrm{E}-20$ & 0.15144 & 0.859 \\
100 & 0 & $9.70 \mathrm{E}+18$ & 10,000 & $8.28 \mathrm{E}-21$ & 0.08028 & 0.923 \\
100 & 0 & $9.70 \mathrm{E}+18$ & 15,000 & $5.73 \mathrm{E}-21$ & 0.05555 & 0.946 \\
100 & 0 & $9.70 \mathrm{E}+18$ & 20,000 & $4.43 \mathrm{E}-21$ & 0.04295 & 0.958 \\
100 & 1 & $1.31 \mathrm{E}+19$ & 5000 & $1.56 \mathrm{E}-20$ & 0.20479 & 0.815 \\
100 & 1 & $1.31 \mathrm{E}+19$ & 10,000 & $8.28 \mathrm{E}-21$ & 0.10856 & 0.897 \\
100 & 1 & $1.31 \mathrm{E}+19$ & 15,000 & $5.73 \mathrm{E}-21$ & 0.07512 & 0.928 \\
100 & 1 & $1.31 \mathrm{E}+19$ & 20,000 & $4.43 \mathrm{E}-21$ & 0.05808 & 0.944 \\
100 & 2 & $1.61 \mathrm{E}+19$ & 5000 & $1.56 \mathrm{E}-20$ & 0.25125 & 0.778 \\
100 & 2 & $1.61 \mathrm{E}+19$ & 10,000 & $8.28 \mathrm{E}-21$ & 0.13318 & 0.875 \\
100 & 2 & $1.61 \mathrm{E}+19$ & 15,000 & $5.73 \mathrm{E}-21$ & 0.09217 & 0.912 \\
100 & 2 & $1.61 \mathrm{E}+19$ & 20,000 & $4.43 \mathrm{E}-21$ & 0.07126 & 0.931 \\
100 & 4 & $2.23 \mathrm{E}+19$ & 5000 & $1.56 \mathrm{E}-20$ & 0.34792 & 0.706 \\
100 & 4 & $2.23 \mathrm{E}+19$ & 10,000 & $8.28 \mathrm{E}-21$ & 0.18443 & 0.832 \\
100 & 4 & $2.23 \mathrm{E}+19$ & 15,000 & $5.73 \mathrm{E}-21$ & 0.12763 & 0.880 \\
100 & 4 & $2.23 \mathrm{E}+19$ & 20,000 & $4.43 \mathrm{E}-21$ & 0.09867 & 0.906 \\
1000 & 0 & $1.28 \mathrm{E}+20$ & 5000 & $1.56 \mathrm{E}-20$ & 1.99329 & 0.136 \\
1000 & 0 & $1.28 \mathrm{E}+20$ & 10,000 & $8.28 \mathrm{E}-21$ & 1.05662 & 0.348 \\
1000 & 0 & $1.28 \mathrm{E}+20$ & 15,000 & $5.73 \mathrm{E}-21$ & 0.73121 & 0.481 \\
1000 & 0 & $1.28 \mathrm{E}+20$ & 20,000 & $4.43 \mathrm{E}-21$ & 0.56532 & 0.568 \\
1000 & 10 & $1.50 \mathrm{E}+20$ & 5000 & $1.56 \mathrm{E}-20$ & 2.33592 & 0.097 \\
1000 & 10 & $1.50 \mathrm{E}+20$ & 10,000 & $8.28 \mathrm{E}-21$ & 1.23824 & 0.290 \\
1000 & 10 & $1.50 \mathrm{E}+20$ & 15,000 & $5.73 \mathrm{E}-21$ & 0.85690 & 0.424 \\
1000 & 10 & $1.50 \mathrm{E}+20$ & 20,000 & $4.43 \mathrm{E}-21$ & 0.66249 & 0.516 \\
1000 & 20 & $1.75 \mathrm{E}+20$ & 5000 & $1.56 \mathrm{E}-20$ & 2.73466 & 0.065 \\
1000 & 20 & $1.75 \mathrm{E}+20$ & 10,000 & $8.28 \mathrm{E}-21$ & 1.44962 & 0.235 \\
1000 & 20 & $1.75 \mathrm{E}+20$ & 15,000 & $5.73 \mathrm{E}-21$ & 1.00318 & 0.367 \\
1000 & 20 & $1.75 \mathrm{E}+20$ & 20,000 & $4.43 \mathrm{E}-21$ & 0.77558 & 0.460 \\
1000 & 40 & $2.34 \mathrm{E}+20$ & 5000 & $1.56 \mathrm{E}-20$ & 3.65359 & 0.026 \\
1000 & 40 & $2.34 \mathrm{E}+20$ & 10,000 & $8.28 \mathrm{E}-21$ & 1.93673 & 0.144 \\
& 40 & $2.34 \mathrm{E}+20$ & 15,000 & $5.73 \mathrm{E}-21$ & 1.34027 & 0.262 \\
10 & $2.34 \mathrm{E}+20$ & 20,000 & $4.43 \mathrm{E}-21$ & 1.03620 & 0.355
\end{tabular}

${ }^{\text {a}}$ Located at the University of Queensland (1991).

\section{Results}

In this work, two specimen chamber pressures were considered-100 $\mathrm{Pa}$ and $1000 \mathrm{~Pa}$ - which are typical values for commercial ESEM operation. For the first case (Fig. 1), the back-pressure at the pump was set to $0 \mathrm{~Pa}$, corresponding to an ideal physical limiting case. The results are given in Table 1 for four accelerating voltages: 5, 10, 15, and $20 \mathrm{kV}$. 
Table 3. Corresponding Values for the Philips XL30 ESEM ${ }^{\mathrm{a}}$

\begin{tabular}{lccrccc}
\hline$p$ & $p_{1}$ & mol_thick & \multicolumn{1}{c}{$\mathrm{E}$} & & & \\
$(\mathrm{Pa})$ & $(\mathrm{Pa})$ & $\left(\right.$ atoms $\left./ \mathrm{m}^{2}\right)$ & \multicolumn{1}{c}{$(\mathrm{eV})$} & $\sigma_{\mathrm{T}}\left(\mathrm{m}^{2}\right)$ & $m$ & $I / I_{0}$ \\
\hline 100 & 0 & $2.105 \mathrm{E}+19$ & 5000 & $1.56 \mathrm{E}-20$ & 0.32875 & 0.720 \\
100 & 0 & $2.105 \mathrm{E}+19$ & 10,000 & $8.28 \mathrm{E}-21$ & 0.17427 & 0.840 \\
100 & 0 & $2.105 \mathrm{E}+19$ & 15,000 & $5.73 \mathrm{E}-21$ & 0.12060 & 0.886 \\
100 & 0 & $2.105 \mathrm{E}+19$ & 20,000 & $4.43 \mathrm{E}-21$ & 0.09324 & 0.911 \\
100 & 1 & $2.336 \mathrm{E}+19$ & 5000 & $1.56 \mathrm{E}-20$ & 0.36496 & 0.694 \\
100 & 1 & $2.336 \mathrm{E}+19$ & 10,000 & $8.28 \mathrm{E}-21$ & 0.19346 & 0.824 \\
100 & 1 & $2.336 \mathrm{E}+19$ & 15,000 & $5.73 \mathrm{E}-21$ & 0.13388 & 0.875 \\
100 & 1 & $2.336 \mathrm{E}+19$ & 20,000 & $4.43 \mathrm{E}-21$ & 0.10351 & 0.902 \\
100 & 2 & $2.705 \mathrm{E}+19$ & 5000 & $1.56 \mathrm{E}-20$ & 0.42249 & 0.655 \\
100 & 2 & $2.705 \mathrm{E}+19$ & 10,000 & $8.28 \mathrm{E}-21$ & 0.22396 & 0.799 \\
100 & 2 & $2.705 \mathrm{E}+19$ & 15,000 & $5.73 \mathrm{E}-21$ & 0.15498 & 0.856 \\
100 & 2 & $2.705 \mathrm{E}+19$ & 20,000 & $4.43 \mathrm{E}-21$ & 0.11982 & 0.887 \\
100 & 4 & $3.318 \mathrm{E}+19$ & 5000 & $1.56 \mathrm{E}-20$ & 0.51824 & 0.596 \\
100 & 4 & $3.318 \mathrm{E}+19$ & 10,000 & $8.28 \mathrm{E}-21$ & 0.27471 & 0.760 \\
100 & 4 & $3.318 \mathrm{E}+19$ & 15,000 & $5.73 \mathrm{E}-21$ & 0.19011 & 0.827 \\
100 & 4 & $3.318 \mathrm{E}+19$ & 20,000 & $4.43 \mathrm{E}-21$ & 0.14698 & 0.863 \\
1000 & 0 & $2.691 \mathrm{E}+20$ & 5000 & $1.56 \mathrm{E}-20$ & 4.20410 & 0.015 \\
1000 & 0 & $2.691 \mathrm{E}+20$ & 10,000 & $8.28 \mathrm{E}-21$ & 2.22855 & 0.108 \\
1000 & 0 & $2.691 \mathrm{E}+20$ & 15000 & $5.73 \mathrm{E}-21$ & 1.54222 & 0.214 \\
1000 & 0 & $2.691 \mathrm{E}+20$ & 20,000 & $4.43 \mathrm{E}-21$ & 1.19233 & 0.304 \\
1000 & 10 & $2.888 \mathrm{E}+20$ & 5000 & $1.56 \mathrm{E}-20$ & 4.51102 & 0.011 \\
1000 & 10 & $2.888 \mathrm{E}+20$ & 10,000 & $8.28 \mathrm{E}-21$ & 2.39124 & 0.092 \\
1000 & 10 & $2.888 \mathrm{E}+20$ & 15,000 & $5.73 \mathrm{E}-21$ & 1.65481 & 0.191 \\
1000 & 10 & $2.888 \mathrm{E}+20$ & 20,000 & $4.43 \mathrm{E}-21$ & 1.27937 & 0.278 \\
1000 & 20 & $3.113 \mathrm{E}+20$ & 5000 & $1.56 \mathrm{E}-20$ & 4.86233 & 0.008 \\
1000 & 20 & $3.113 \mathrm{E}+20$ & 10,000 & $8.28 \mathrm{E}-21$ & 2.57747 & 0.076 \\
1000 & 20 & $3.113 \mathrm{E}+20$ & 15,000 & $5.73 \mathrm{E}-21$ & 1.78368 & 0.168 \\
1000 & 20 & $3.113 \mathrm{E}+20$ & 20,000 & $4.43 \mathrm{E}-21$ & 1.37901 & 0.252 \\
1000 & 40 & $3.724 \mathrm{E}+20$ & 5000 & $1.56 \mathrm{E}-20$ & 5.81746 & 0.003 \\
1000 & 40 & $3.724 \mathrm{E}+20$ & 10,000 & $8.28 \mathrm{E}-21$ & 3.08378 & 0.046 \\
& 40 & $3.724 \mathrm{E}+20$ & 15,000 & $5.73 \mathrm{E}-21$ & 2.13406 & 0.118 \\
& 40 & $3.724 \mathrm{E}+20$ & 20,000 & $4.43 \mathrm{E}-21$ & 1.64990 & 0.192
\end{tabular}

${ }^{a}$ Located at the University of Technology, Sydney (1999).

Since the back-pressure at the gas exit interface is not exactly known for the two commercial instruments, four pressure values were chosen over a pressure range in which the real pressure is expected to fall; the back-pressure is referred to as "pump pressure." These values were $0,1,2$, and $4 \mathrm{~Pa}$ when the specimen chamber pressure was $100 \mathrm{~Pa}$, and $0,10,20$, and $40 \mathrm{~Pa}$ when the specimen chamber pressure was $1000 \mathrm{~Pa}$. The pressure at the second PLA interface at the high-vacuum interface was assumed to be zero in all

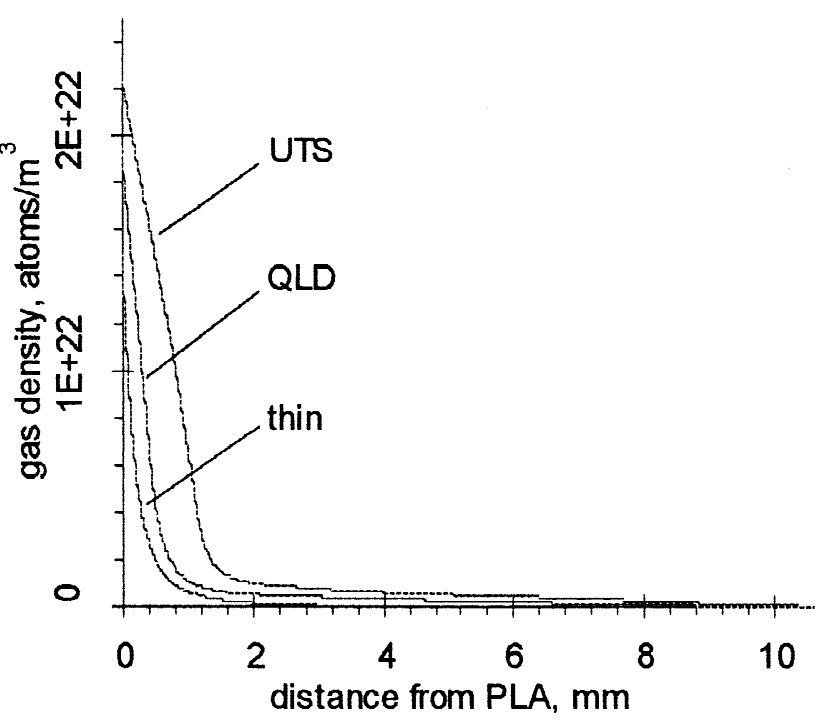

Figure 4. Argon at $100 \mathrm{~Pa}$. Density variation in gas downstream of the PLA plane along the axis for the thin, ElectroScan E3 (1991), located at the University of Queensland (QLD), and XL30 ESEM (1999), located at the University of Technology, Sydney (UTS), starting at $100 \mathrm{~Pa}$ in the upstream specimen chamber.

cases. The beam current loss was determined again at four accelerating voltages, 5, 10, 15, and $20 \mathrm{kV}$ for each of the eight pressure combinations. The results are presented in Table 2 for the E3 ESEM instrument and in Table 3 for the XL30 ESEM instrument.

Graphical representations of some of the data are shown in Figures 4-10. Figure 4 shows the variation in gas particle density (atoms per cubic meter) along the axis of the three systems, when the specimen chamber is held at $100 \mathrm{~Pa}$ and the pump pressure is $0 \mathrm{~Pa}$. Each curve represents the average density of four radial positions for each point along the axis, namely, at $0.05,0.10,0.15$, and $0.20 \mathrm{~mm}$, where the density varies slightly.

Similarly, the density variation along the jet axis for the three systems when the specimen chamber pressure is 1000 $\mathrm{Pa}$ and the pump pressure is $0 \mathrm{~Pa}$ is illustrated in Figure 5. For the variation in gas density along the axis of the E3 ESEM system, shown in Figures 6 and 7, the specimen chamber was held at $100 \mathrm{~Pa}$ and $1000 \mathrm{~Pa}$, respectively, at the chosen pump pressures. For comparison, the ideal-case curve (thin aperture at $0 \mathrm{~Pa}$ pump pressure) is also reproduced. The corresponding results for the XL30 ESEM are presented in Figures 8 and 9.

Finally, a graphical representation of the transmitted fraction of a $5 \mathrm{kV}$ electron beam into the specimen chamber 


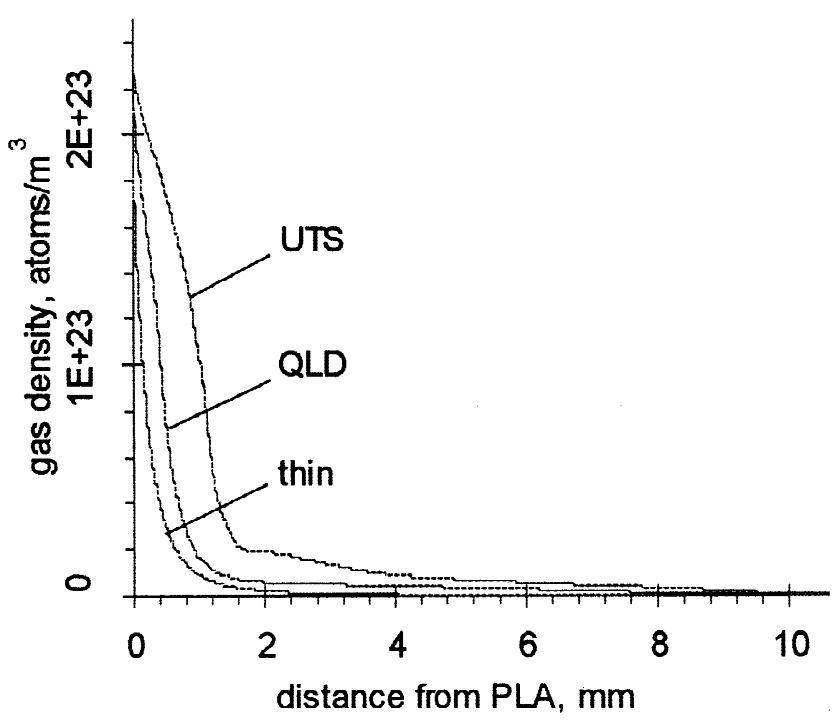

Figure 5. Argon at $1000 \mathrm{~Pa}$. Density variation in gas downstream of the PLA plane along the axis for the thin, ElectroScan E3 (1991), located at the University of Queensland, and the XL30 ESEM (1999), located at the University of Technology, Sydney, starting at $1000 \mathrm{~Pa}$ in the upstream specimen chamber.

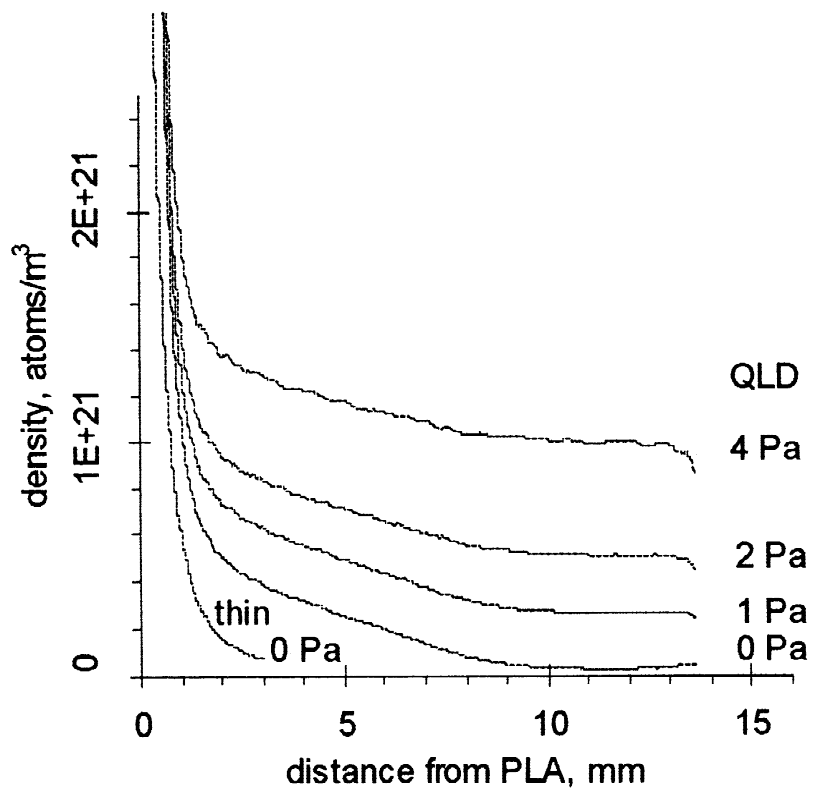

Figure 6. Argon at $100 \mathrm{~Pa}$. Density variation in gas downstream of the PLA plane for the ElectroScan E3 (1991), located at the University of Queensland, at four pump pressures, with $100 \mathrm{~Pa}$ in the specimen chamber. The thin case is reproduced from Figure 4.

is shown in Figure 10 for three cases: the fraction is given for all three microscopes with specimen chamber at either 100 or $1000 \mathrm{~Pa}$, and $0 \mathrm{~Pa}$ pump pressure, corresponding to an infinite pumping speed.

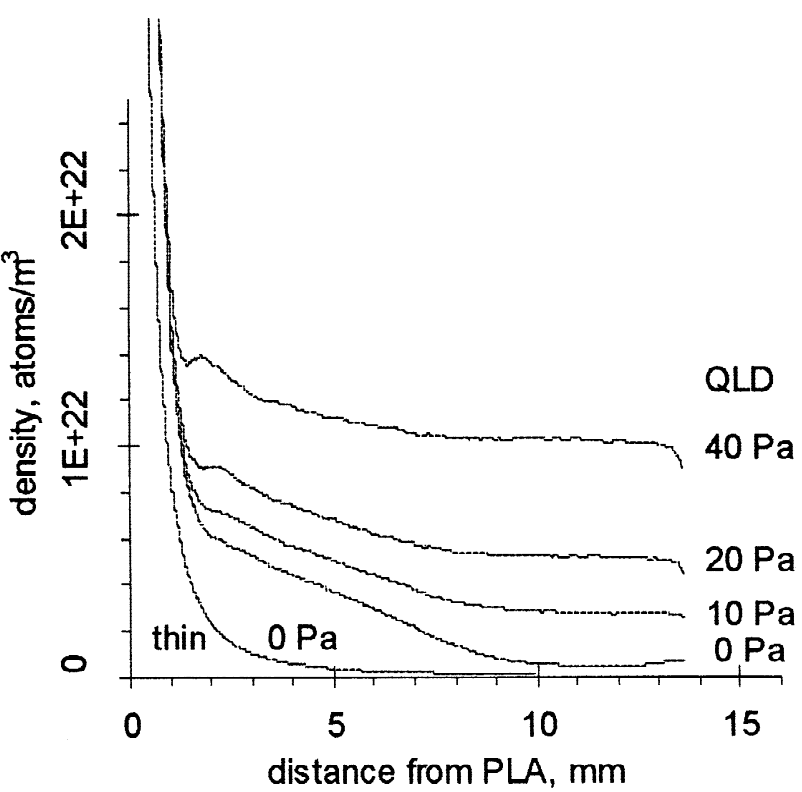

Figure 7. Argon at $1000 \mathrm{~Pa}$. Density variation in gas downstream of the PLA plane for the ElectroScan E3 (1991), located at the University of Queensland, at four pump pressures, with $1000 \mathrm{~Pa}$ in the specimen chamber. The thin case is reproduced from Figure 5.

\section{Discussion}

This work has established that a thin-plate PLA assembly produces the minimum electron beam current loss at the high-vacuum - high-pressure boundary. This result was expected because the thin PLA walls result in the most abrupt change in pressure, which generates the fastest gas density decrease along the axis of the gas jet. The thicker PLA design employed in the commercial ESEMs creates a slower pressure transition, which leads to greater loss in beam current above the PLA.

With the thin PLA there is sufficient electron current in the probe left for imaging using all of the accelerating voltages and pressures evaluated in this study (see Table 1). Gases with a lower scattering cross section, such as water vapor and nitrogen, should also form high-quality images at higher pressure and, indeed, good-quality images have been recorded at pressures much greater than $1000 \mathrm{~Pa}$ at $5 \mathrm{kV}$ (Danilatos, 1988).

Tables 2 and 3 illustrate that both commercial instruments provide adequate beam current at $100 \mathrm{~Pa}$ specimen chamber pressure, but significant beam current loss results when the pressure is raised above $1000 \mathrm{~Pa}$. It is clear from Figures 7 and 9 that pump efficiency pressure plays an important role in instrument design and performance. As 


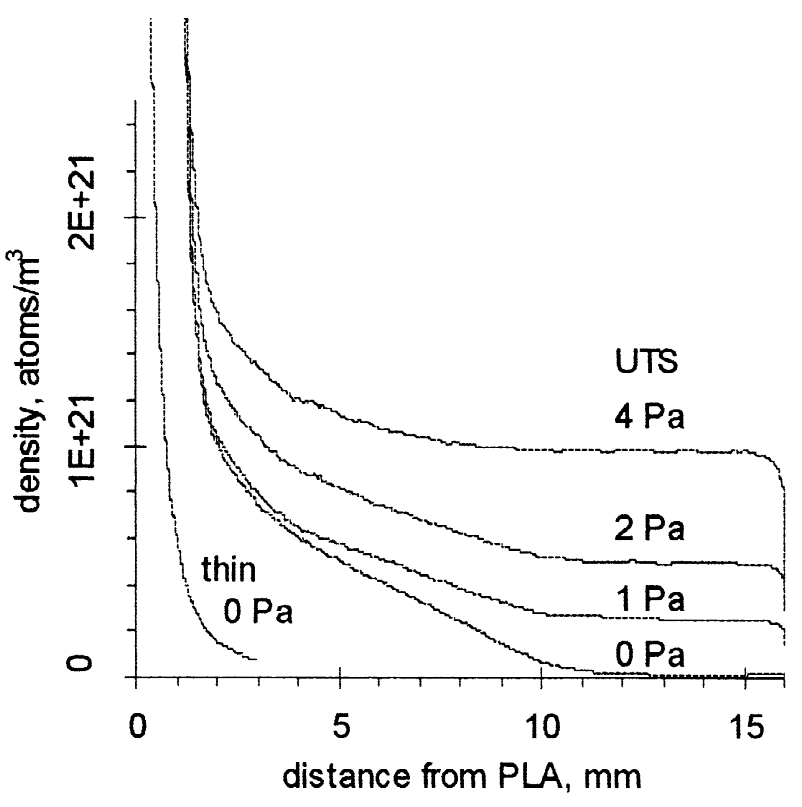

Figure 8. Argon at $100 \mathrm{~Pa}$. Density variation in gas downstream of the PLA plane for the XL30 ESEM, located at the University of Technology, Sydney, at four pump pressures, with $100 \mathrm{~Pa}$ in the specimen chamber. The thin case is reproduced from Figure 4.

the pump pressure increases from 0 to $40 \mathrm{~Pa}$, a significant component of molecular thickness is added and a considerable increase in beam current loss results. The area under each curve in these figures represents the molecular thickness; the deviation from the ideal case is clear.

A comparative presentation of the transmitted fraction of a $5 \mathrm{kV}$ electron beam at $100 \mathrm{~Pa}$ and $1000 \mathrm{~Pa}$ chamber pressures is also shown in Figure 10 for all three systems operating under ideal pumping conditions, namely with a 0 Pa pump pressure. The results clearly demonstrate that there is a significant difference between an earlier and recent model of commercial ESEM; this is due to the different designs between bullets. Furthermore, there is a significant difference between the commercial ESEM instruments and a prototype ESEM using a thin PLA geometry.

The actual electron beam current loss for any XL30 or E3 ESEM (using bullets designs similar to those studied in this work) can be determined by accurately measuring the pump pressure and interpolating the data given here. However, care should be taken to ensure that proper pressure gauge calibration procedures are followed when using different gases (e.g., argon in this case).

To accurately find the effect of electron beam current loss above the PLA with other imaging gases, the DSMC simulation procedure must be repeated for each gas sepa-

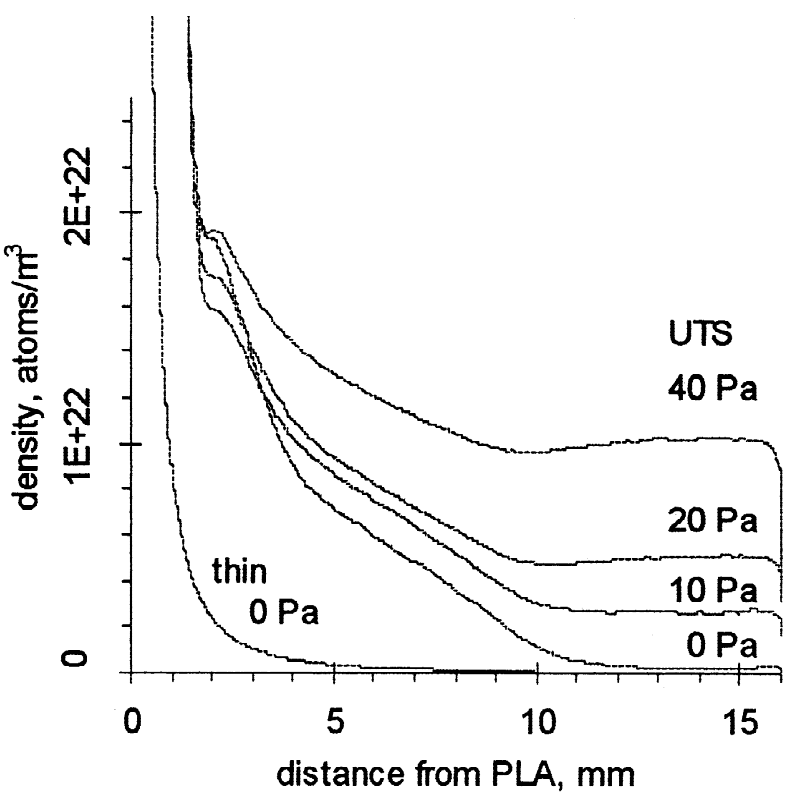

Figure 9. Argon at $1000 \mathrm{~Pa}$. Density variation in gas downstream of the PLA plane for the XL30 ESEM, located at the University of Technology, Sydney, at four pump pressures, with $1000 \mathrm{~Pa}$ in the specimen chamber. The thin case is reproduced from Figure 5.

rately. However, the results presented here may be used to approximate the electron beam current loss with other gases by simply applying the appropriate scattering cross sections to the molecular thickness tabulated here.

The electron beam current loss above the PLA can be reduced in the commercial ESEM by increasing the operating voltage of the ESEM. However, this is not desirable in many applications, especially with organic and insulating specimens, because high accelerating voltages result in large beam-specimen interaction volumes (or beam penetration) and the injection of large amounts of charge, which usually results in specimen damage. A large interaction volume also severely limits the resolution in backscattered electron mode. Another approach to minimize the beam current loss is to use a smaller-diameter PLA aperture, which will reduce the gas flow and the overall molecular thickness and reduce beam current loss above the PLA. However, this solution seriously restricts the field of view at low magnifications, which may be undesirable for many applications.

Electron beam current loss above the PLA can be significantly reduced in the commercial ESEMs investigated in this work by simply incorporating an optimum (thin) PLA into their differential pumping system. In addition to a thin-plate PLA investigated in this study, a conical geometry has also been evaluated by Danilatos (1993). The determi- 


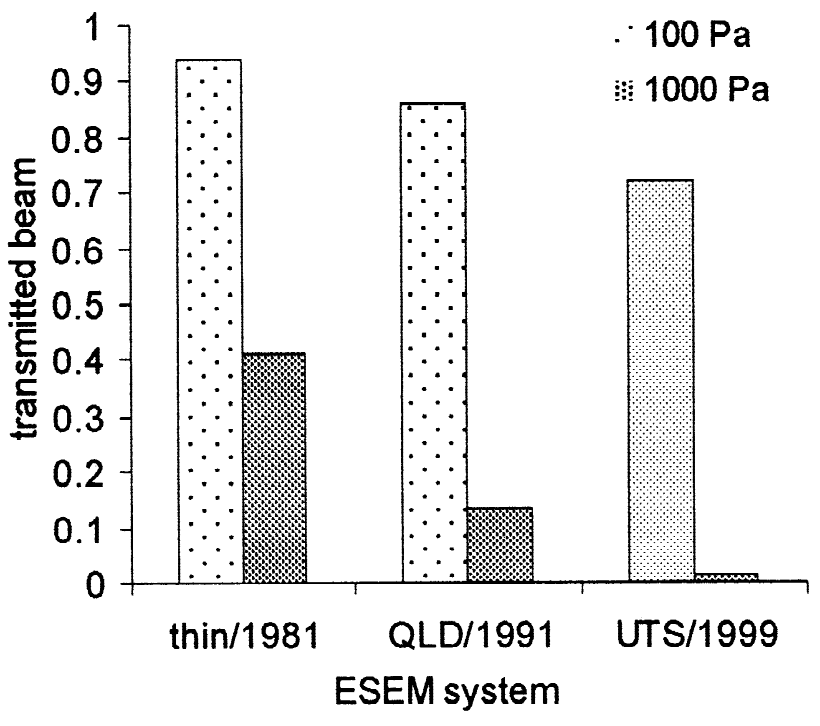

Figure 10. Transmitted fraction of a $5 \mathrm{kV}$ argon electron beam through the PLAs of the prototype ESEM (thin), ElectroScan E3 (1991), located at the University of Queensland, and the XL30 ESEM (1999), located at the University of Technology, Sydney, at chamber pressures of $100 \mathrm{~Pa}$ and $1000 \mathrm{~Pa}$, with pump pressure at $0 \mathrm{~Pa}$.

nation of optimum thickness, cone angle, distance between the two PLA, and the interplay of these parameters will be the subject of future reporting.

A recent study by Danilatos (2000) has shown that it is possible to overcome even the natural limit posed by the gas jet if a reverse-flow PLA (RF-PLA) is used. With an RFPLA, an annular supersonic gas jet is introduced in the opposite direction, toward the specimen chamber, around the PLA, with a pumping action at its core. The conventional gas jet above the PLA is eliminated. As a result, the electron beam effectively does not suffer loss above the PLA.

Another consideration is the distribution of electrons lost (or removed) from the electron beam into what has been termed the "electron skirt." This is particularly important in X-ray microanalysis (Bolon, 1991; Griffin, 1992; Doehne, 1997). Study of the electron skirt can be done experimentally, theoretically, and computationally. All previous studies assume an abrupt or step-wise function of the gas density, where the electron beam traverses a uniform gas layer from the PLA plane to the specimen plane. However, the present study has shown that at high chamber pressures and low operating voltages, there is a significant gas density above the PLA, which results in a significant electron skirt forming prior to the beam entering the specimen chamber.
Future computation of the intensity and distribution of the electron skirt incident on a specimen surface should take into account the electron scattering in the gas jet above the PLA.

The depletion of gas immediately below the PLA and the possible perturbation of flow by the specimen shape and position should also be considered in future work. When such perturbation occurs, the pressure reading for the specimen chamber can be significantly different from the actual pressure prevailing over the examined surface, which can easily provide misleading results. The DSMC method provides the density at every point in the entire gas flow field, which can be used as input to calculations of electron skirt distributions and other studies.

\section{CONCLUSIONS}

The conventional differential pumping system is characterized by a supersonic gas plume formed downstream of a PLA (i.e., above the PLA in the "vacuum" part of the column). This gas represents a mass thickness, which the electron beam of an ESEM must overcome, before it enters in the specimen chamber. The mass thickness results in a certain amount of electron beam current loss. The gas density variation in the jet has been computed by the DSMC method. These computations have been made for a thinplate PLA, which represents a natural limit of mass thickness. Two commercial ESEM instruments have also been studied and found to incur much greater electron beam losses because of the thicker PLA employed in these microscopes. As a consequence, these instruments may experience operational difficulties due to the lack of probe current when an accelerating voltage below $5 \mathrm{kV}$ is used with argon gas pressures $>1000 \mathrm{~Pa}$ in the specimen chamber. However, an optimum PLA design should allow operation under these conditions. These results show that quantitative studies to determine optimum conventional differential pumping systems can be done with the DSMC method.

\section{REFERENCES}

Bird GA (1995) Molecular Gas Dynamics and the Direct Simulation of Gas Flows. Oxford: Oxford Science Publications, Clarendon Press 
Bolon RB (1991) X-ray Microanalysis in the ESEM. In: Microbeam Analysis - 1991, Howitt, DG (ed). San Francisco: San Francisco Press, pp 199-200

Danilatos GD (1981) Design and construction of an atmospheric or environmental SEM (part 1). Scanning 4:9-20

Danilatos GD (1983) Design and construction of an atmospheric or environmental SEM-2. Micron 14:41-52

Danilatos GD (1985) Design and construction of an atmospheric or environmental SEM (part 3). Scanning 7:26-42

Danilatos GD (1988) Foundations of environmental scanning electron microscopy. Adv Electronics Electron Phys 71:109-250

Danilatos GD (1990) Theory of the gaseous detector device in the environmental scanning electron microscope. Adv Electronics Electron Phys 78:1-102

Danilatos GD (1991) Gas flow properties in the environmental
SEM. In: Microbeam Analysis_-1991 Howitt DG (ed). San Francisco: San Francisco Press, pp 201-203

Danilatos GD (1993) Environmental scanning electron microscope: some critical issues. Scan Microsc Suppl 7:57-80

Danilatos GD (2000) Reverse flow pressure limiting aperture (RFPLA). Microsc Microanal 6:21-30

Doehne E (1997) A new correction method for high-resolution energy-dispersive X-ray analyses in the environmental scanning electron microscope. Scanning 19:75-78

Griffin BJ (1992) Effects of chamber pressure and accelerating voltage on X-ray resolution in the ESEM. In: Proceedings of the 50th Annual Meeting of the Electron Microscopy Society of America, Bailey, Bentley, Small (eds). pp 1324-1325

Jost K, Kessler J (1963) Die Ortsverteilung mittelschneller Elektronen bei Mehrfachstreung. Z Phys 176:126-142 